\title{
A novel method for evaluating correlation functions in lattice hadron spectroscopy*
}

\author{
Justin Foley; Chik Him Wong \\ Dept. of Physics, Carnegie Mellon University, Pittsburgh, PA 15213, USA \\ E-mail: jfoley@physics.utah.edu, chikhimw@andrew.cmu.edu
}

John Bulava

NIC, DESY, Platanenallee 6, 15738, Zeuthen, Germany

\section{K. Jimmy Juge}

Dept. of Physics, University of the Pacific, Stockton, CA 95211, USA

\section{David Lenkner, Colin Morningstar}

Dept. of Physics, Carnegie Mellon University, Pittsburgh, PA 15213, USA

\section{Michael Peardon}

School of Mathematics, Trinity College, Dublin 2, Ireland

\begin{abstract}
We describe a new approach for evaluating hadronic correlation functions which combines Laplacian-Heaviside quark smearing with a stochastic estimator of quark propagators. This method utilizes noise dilution in a new way to reduce the variance in correlators. The efficacy of the new algorithm is demonstrated on a number of systems, including disconnected diagrams and multi-hadron correlators, on a small lattice where comparisons with the results obtained with exactly determined quark propagators are possible. On larger lattice volumes, the use of exact propagators becomes prohibitively expensive, while the stochastic method is still computationally feasible.
\end{abstract}

The XXVIII International Symposium on Lattice Field Theory, Lattice2010

June 14-19, 2010

Villasimius, Italy

\footnotetext{
* Based on talks given by Justin Foley and Chik Him Wong.

${ }^{\dagger}$ Present address: Dept. of Physics at the University of Utah, and The Center for Computational Sciences, University of Tsukuba.
} 


\section{Introduction}

A long-term aim of our collaboration is the first-principles determination of stable hadron masses, and resonance energies and decay widths. To date, considerable progress has been made in extracting finite-volume stationary-state energies in the isovector meson, kaon, and baryon sectors $[1,2,3]$. These initial studies, which were performed at relatively heavy pion masses and moderate lattice volumes, involved interpolating operators specifically designed to couple strongly to single-hadron states. However, at lighter pion masses and larger spatial volumes, explicit multihadron operators must be incorporated into the analysis. Until recently, the inclusion of multihadron interpolators has been problematic because the resulting two-point functions may involve quark lines which begin and end on the sink time slice and source operators on all spatial sites of a time slice. Hence, a general treatment of multi-hadrons is not amenable to conventional pointto-all quark-propagator techniques. In analyses involving single-particle operators, disconnected contributions to flavor-singlet meson correlators are similarly problematic, and little progress has been made to date in determining the $I=0$ meson spectrum from Monte Carlo simulations.

In this article, we describe a novel method [4] for evaluating hadronic correlation functions, which combines quark-field smearing with a stochastic estimator. The new method facilitates the precise evaluation of multi-hadron and flavor-singlet meson correlators, at a significantly lower computational cost than previous approaches. First results obtained on intermediate lattice volumes are presented, and we briefly discuss more recent studies performed on larger lattices.

\section{An alternate quark-field smearing scheme}

Our approach relies on the fact that generally in a spectroscopy calculation, gauge-covariant smearing is applied to the quark fields in hadron interpolators in order to reduce the coupling of these operators to very high-lying states. In the resulting correlation functions, the quark propagator $M^{-1}$ is always sandwiched between smearing operators: $S M^{-1} S$, where $\tilde{\psi}=S \psi$ is the smeared quark field. We define Laplacian-Heaviside [5], or LapH, quark-field smearing by

$$
S=\Theta\left(\tilde{\triangle}+\sigma^{2}\right), \quad S_{a b}(x, y) \approx \delta_{x_{4}, y_{4}} \sum_{k=1}^{N_{v}} v_{a}^{(k)}(x) v_{b}^{(k)}(y)^{*}
$$

with

$$
\tilde{\triangle}_{a b}(x, y) v_{b}^{(i)}(y)=-\lambda_{i}\left(x_{4}\right) v_{a}^{(i)}(x), \quad v^{(i) *}(x) v^{(j)}(x)=\delta^{i j},
$$

where $\tilde{\triangle}$ is a gauge-covariant Laplacian operator constructed from stout-smeared [6] link variables $\tilde{U}$ :

$$
\tilde{\triangle}_{a b}(x, y)=\sum_{k=1}^{3}\left\{\tilde{U}_{k}^{a b}(x) \delta(x+\hat{k}, y)+\tilde{U}_{k}^{\dagger a b}(y) \delta(x-\hat{k}, y)-2 \delta(x, y) \delta^{a b}\right\}
$$

The use of smeared link variables in the quark-field smearing operator has been shown to significantly reduce the variance in hadronic correlation functions [7]. The eigenvalues of the Laplacian operator, $-\lambda_{i}$, are negative (see Fig. 1), and the Heaviside function in Eq. 2.1 imposes a cutoff 

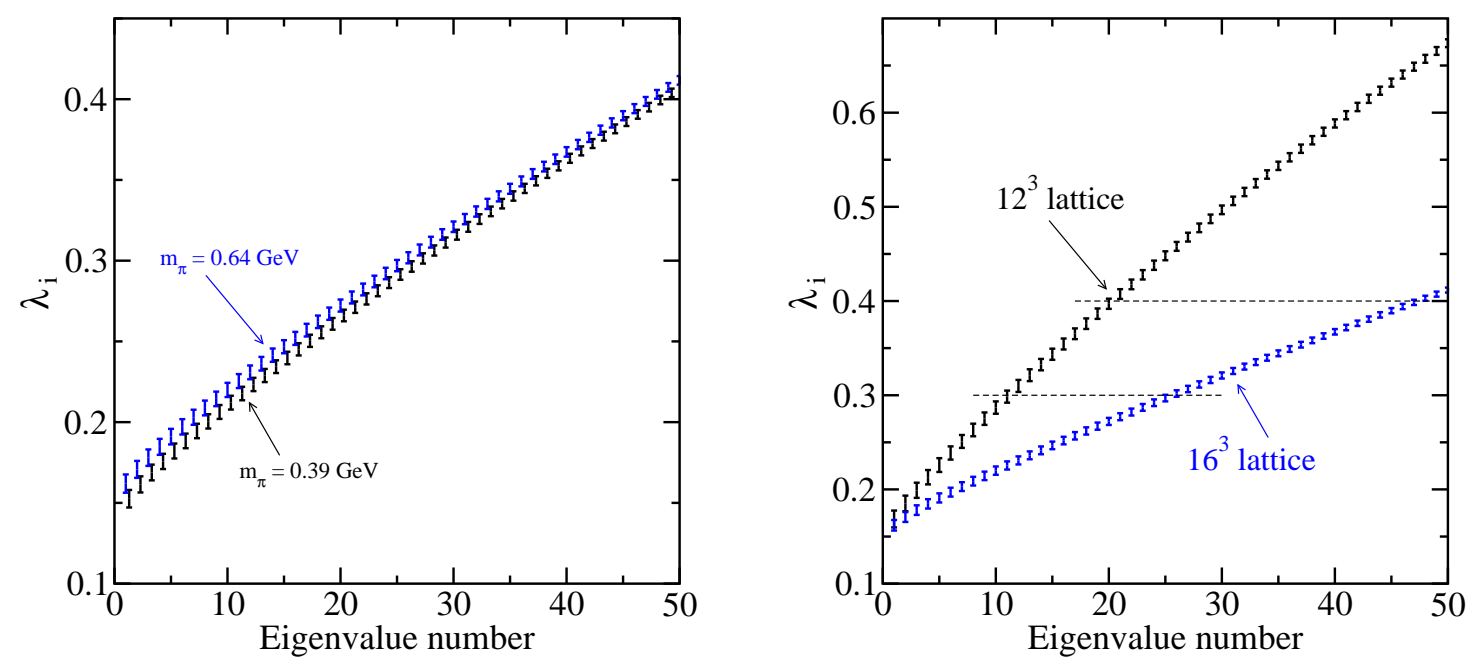

Figure 1: The plot on the left shows the Monte Carlo estimate of low-lying $\lambda_{i}$ 's computed on a $16^{3}$ spatial lattice on dynamical ensembles with two different pion masses using stout-smeared link variables. The pionmass dependence of the Laplacian spectrum is seen to be very mild. The right-hand plot shows Laplacian spectra computed on $2+1$ flavors simulations performed on two different lattice volumes. All other simulation parameters match. The dashed horizontal lines are guides for the eye, showing that the density of eigenmodes grows linearly with the spatial lattice volume.

(parametrized by $\sigma^{2}$ ) on high-momentum modes. The level of quark smearing is increased by lowering the cutoff, thereby excluding more Laplacian eigenmodes. To evaluate hadronic correlation functions involving LapH-smeared quark fields, one does not need to compute the quark-propagator components directly, instead only the matrix elements of the propagator between a subset of Laplacian eigenvectors are required. For a high enough level of quark smearing, i.e., a small enough subset of eigenvectors, it is feasible to compute hadron correlation functions which are currently beyond the reach of conventional lattice techniques [5]. In practice, however, the volume dependence of the Laplacian eigenmode distribution is a significant limitation when computing $S M^{-1} S$ exactly. Although the quark-mass dependence of the Laplacian spectrum is mild, the density of eigenmodes scales linearly with the spatial lattice volume. Therefore, while an exact treatment of $S M^{-1} S$ may work well on smaller lattice volumes as it stands, the increased density of eigenmodes makes this approach impractical on larger lattices.

\subsection{Stochastic estimation}

To mitigate the volume dependence, the LapH smearing scheme can be combined with a stochastic estimator. To proceed, we write

$$
S M^{-1} S=S M^{-1}|v\rangle E\left(\eta \eta^{\dagger}\right)\langle v| .
$$

$E\left(\eta \eta^{\dagger}\right)$ denotes an expectation value over the outer product of noise vectors whose components satisfy

$$
E\left(\eta_{i \alpha}(t)\right)=0, \quad E\left(\eta_{i \alpha}(t) \eta_{j \beta}^{*}\left(t^{\prime}\right)\right)=\delta_{i j} \delta_{\alpha \beta} \delta_{t t^{\prime}}
$$


where $i(j)$ and $\alpha(\beta)$ are eigenmode and spin indices, respectively. Note that the noise vectors have neither color nor spatial indices. The number of quark-matrix inversions required to estimate the smeared quark line in this way is determined by the number of stochastic vectors employed rather than the number of Laplacian eigenvectors used in the smearing. However, in general, this naive estimate for the smeared quark line is too noisy to be of practical use, and we must apply a variance reduction scheme to obtain an improved stochastic estimate.

In a number of systems, noise partitioning, or dilution, turns out to be a particularly effective variance reduction technique [8]. Dilution is implemented by choosing noise vectors whose components have unit norm, i.e., $Z_{n}$ or $U(1)$ noise, and partitioning the noise vector indices, eigenmode, time and spin, into disjoint sets. To each set of indices, $d$, we assign a projection operator $P^{[d]}$ which acts on the noise vectors, with components

$$
\begin{aligned}
& P_{i j \alpha \beta}^{[d]}\left(t, t^{\prime}\right)=1 \text { if }(t, i, \alpha) \text { and }(t, j, \beta) \in d, \\
& P_{i j \alpha \beta}^{[d]}\left(t, t^{\prime}\right)=0 \text { otherwise. }
\end{aligned}
$$

The naive noise average $E\left(\eta \eta^{\dagger}\right)$ is replaced by an expectation value involving diluted noise vectors $\sum_{d} E\left(\eta^{[d]} \eta^{[d] \dagger}\right)$, where $\eta^{[d]}=P^{[d]} \eta$. In the maximal dilution limit, the exact smearing scheme is recovered. We refer to the combination of the LapH smearing scheme with a dilute stochastic estimator as the Stochastic LapH method.

\section{Implementation}

In practice, the estimates for the smeared quark propagator are implemented in terms of pseudofermion fields which we call quark-line ends. For each diluted noise vector $\eta^{[d]}$, we form a quarkline source $\rho^{[d]}$, with components

$$
\rho_{\alpha a}^{[d]}(x)=\sum_{i} \eta_{i \alpha}^{[d]}\left(x_{4}\right) v_{i a}(x)
$$

For each source field, there is a corresponding quark-line sink $\phi^{[d]}=S M^{-1} \rho^{[d]}$. The smeared quark line can then be written

$$
S M^{-1} S=\sum_{d} E\left(\phi^{[d]} \rho^{[d] \dagger}\right)
$$

In addition, the fact that the quark propagator satisfies

$$
M^{-1}(y ; x)=\gamma_{5}\left[M^{-1}(x ; y)\right]^{\dagger} \gamma_{5}
$$

and the hermiticity of the quark smearing operator lead to a second expression for the smeared quark line

$$
S M^{-1} S=\sum_{d} E\left(\bar{\rho}^{[d]} \bar{\phi}^{[d] \dagger}\right)
$$

where $\bar{\rho}^{[d]}=\gamma_{5} \rho^{[d]}$ and $\bar{\phi}^{[d]}=\gamma_{5} \phi^{[d]}$. 
On a typical lattice, the complete set of pseudofermion fields needed to implement the quarkline estimate is too large to save to disk. Instead, to store the quark-line estimates on a given configuration, we save the set of low-lying Laplacian eigenvectors used in the smearing, the diluted noise vectors $\eta^{[d] 1}$, and, for each noise vector, a tensor with elements

$$
\Pi\left(i, \alpha, x_{4} ; d\right)=\sum_{\mathbf{x} a} v_{i a}^{*}(x) \phi_{\alpha a}^{[d]}(x) .
$$

Using these components, the pseudofermion source and sink fields can be quickly reconstructed when needed. Only the Laplacian eigenvectors require a significant amount of storage, which is however independent of the number of quark lines to be estimated and the number of noise vectors and the dilution schemes used.

To illustrate how the quark-line ends can be combined to form a hadron correlator, consider the simple meson two-point function

$$
C^{m n}(t)=\left\langle 0\left|\tilde{\psi} \Omega_{m} \tilde{\psi}(t) \tilde{\psi} \Omega_{n}^{\dagger} \tilde{\psi}(0)\right| 0\right\rangle,
$$

where $\Omega_{m}\left(\Omega_{n}\right)$ is an arbitrary combination of gauge-covariant spatial displacement operators and spin matrices acting on the quark fields. For compactness, all indices apart from the time label have been suppressed. Wick contracting the quark fields, and using both Eq. 3.2 and Eq. 3.4, we obtain an estimate for the connected component of this correlator

$$
C_{\text {conn }}^{m n}(t) \approx-\left\langle\frac{1}{N_{r} N_{s}} \sum_{r \neq s} \sum_{d_{r} d_{s}}\left[\bar{\phi}_{r}^{\left[d_{r}\right] \dagger} \Omega_{m} \phi_{s}^{\left[d_{s}\right]}(t) \rho_{s}^{\left[d_{s}\right] \dagger} \Omega_{n}^{\dagger} \bar{\rho}_{r}^{\left[d_{r}\right]}(0)\right]\right\rangle,
$$

where the superscripts $r$ and $s$ label different noise sources and $\langle\ldots\rangle$ denotes an average over the gauge ensemble. Other estimates for the connected correlator, involving different combinations of 'barred' and 'unbarred' quark line ends, are also possible. However, since Eq. 3.7 involves the quark sources $\rho_{r}^{\left[d_{r}\right]}, \rho_{s}^{\left[d_{s}\right]}$ on a single time slice, $t=0$, only, it has the advantage that both quark-line estimates are automatically fully diluted in time.

Defining the mesonic 'operators' $\mathscr{O}_{\Omega_{m}[\bar{r} s]}^{\left[d_{r} d_{s}\right]} \equiv \bar{\phi}_{r}^{\left[d_{r}\right] \dagger} \Omega_{m} \phi_{s}^{\left[d_{s}\right]}$ and $\overline{\mathscr{O}}_{\Omega_{n}[\bar{r} s]}^{\left[d_{r} d_{s}\right]} \equiv\left(\bar{\rho}_{r}^{\left[d_{r}\right] \dagger} \Omega_{n} \rho_{s}^{\left[d_{s}\right]}\right)^{\dagger}=$ $\rho_{s}^{\left[d_{s}\right] \dagger} \Omega_{n}^{\dagger} \bar{\rho}_{r}^{\left[d_{r}\right]}$ allows the connected correlator to be written in a more compact form

$$
C_{\mathrm{conn}}^{m n}(t) \approx-\left\langle\frac{1}{N_{r} N_{s}} \sum_{r \neq s} \sum_{r=d_{r} d_{s}} \mathscr{O}_{\Omega_{m}[\bar{r} s]}^{\left[d_{r} d_{s}\right]}(t) \overline{\mathscr{O}}_{\Omega_{n}[\bar{r} s]}^{\left[d_{r} d_{s}\right]}(0)\right\rangle .
$$

The factorization of the correlator estimates into contributions from different hadron operators is an extremely useful feature of the method. It facilitates the construction of correlator matrices involving large sets of interpolating operators and simplifies the evaluation of multi-hadron correlators.

\section{First tests}

Tests of the Stochastic LapH algorithm on connected hadron correlators, such as isovectormeson and baryon two-point functions, have shown that, with a judiciously chosen dilution scheme,

\footnotetext{
${ }^{1}$ In practice, we simply record a random initial seed, the dilution scheme, and a rule for generating the noise vector from the seed.
} 
this method yields statistical errors that are comparable to the errors obtained using an exact treatment of $S M^{-1} S$, at a considerably lower computational cost. Moreover, the new method is more efficient than using diluted stochastic estimates with noises introduced on the entire lattice, as described in Ref. [8]. This is clearly demonstrated in Fig. 2, which shows the ratios of standard deviation on a single time slice of a nucleon correlator evaluated using different stochastic methods to that obtained from exact LapH. The solid symbols denote the results obtained by introducing noise on the lattice. The open symbols are results from the Stochastic LapH method. The nucleon operator in question has gauge-covariant displacements in three directions. These measurements were performed on $1002+1$ flavor gauge-field configurations with a pion mass of approximately $400 \mathrm{MeV}$, on a $20^{3} \times 128$ anisotropic lattice. The spatial lattice spacing was approximately $0.12 \mathrm{fm}$, and the ratio of spatial to temporal lattice spacings was 3.5. The smearing uses the 64 lowest-lying Laplacian eigenmodes on each time slice. The statistical uncertainties on the correlators were estimated using the Jackknife algorithm. The data here correspond to a temporal separation between the source and sink operators of $t=5 a_{t}$, although the same qualitative behavior is observed for other temporal separations. The minimal level of dilution included in this figure is full time dilution. The reason is that for a connected correlator evaluated on a single source time slice, the inversion cost is the same regardless the time dilution scheme. Any non-full time dilution schemes would only impose larger variance from the noises without any reduction of computational costs. The eigenmode dilution schemes considered include partitioning the Laplacian eigenmodes into blocks of adjacent modes, as well as choosing subsets consisting of eigenmodes that are separated by some number $n$ of intermediate modes, known as interlacing. The Stochastic LapH estimates use three independent $Z_{4}$ noise vectors - the minimum number required to obtain unbiased stochastic estimates. It is possible to improve the correlator estimates by averaging over different orderings of the noise vectors. However, this average has not been performed on the examples presented here.

For a given number of quark-matrix inversions, the error on the correlator computed using Stochastic LapH is much smaller than the error on the correlator estimate involving stochastic quark propagators. Fig. 2 also shows that there is no significant difference between using blocked eigenmode dilution projectors or interlaced eigenmode projectors.

Fig. 3 compares the same error ratios for Stochastic LapH estimates of the nucleon correlator on two different spatial lattice volumes. Results for the $20^{3}$ spatial volume are plotted together with results from a smaller, $16^{3}$ lattice. 32 Laplacian eigenvectors are used to smear the quark field on the $16^{3}$ volume, such that eigenvalue cutoffs on the two lattices are approximately the same. All other input parameters are kept fixed. Increasing the spatial lattice volume for a fixed eigenvalue cutoff and dilution scheme does lead to some increase in the statistical error. However, this increase is modest for all dilution schemes considered and the difference in errors between the two volumes decreases with higher levels of dilution.

\section{Disconnected and multi-hadron correlators}

While the results presented for the connected hadron correlators are promising, the true test of the Stochastic LapH method lies in its application to disconnected diagrams and multi-hadron correlators. In this section, we compare results obtained for these systems using both the Stochastic and exact LapH schemes. Due to the large number of quark-matrix inversions required to imple- 


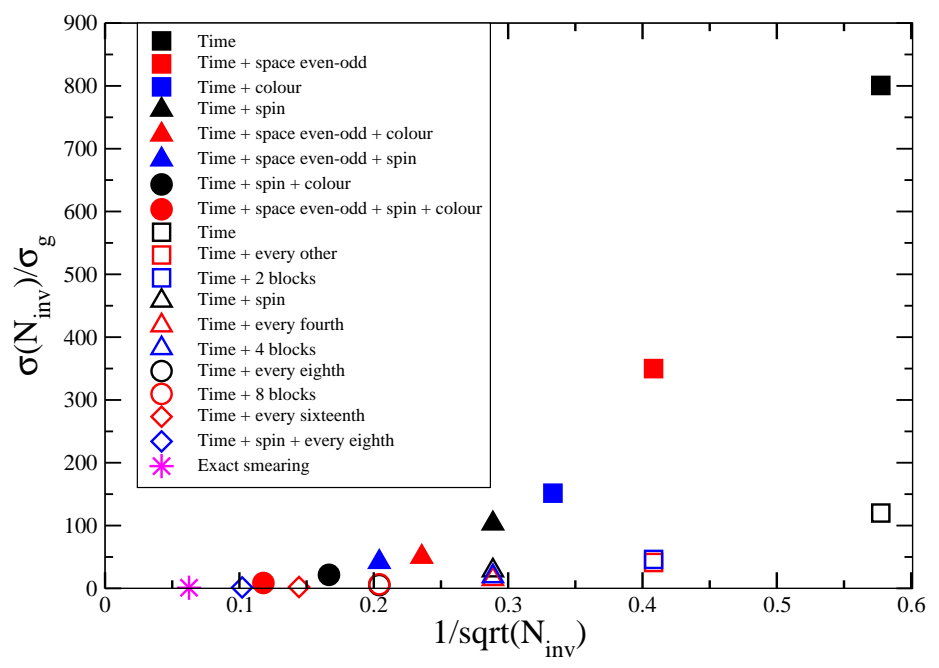

Figure 2: Ratio of standard deviation on time slice 5 of a triply-displaced nucleon correlator evaluated using different stochastic estimators to the standard deviation on the exact LapH estimate of the correlator at the same time separation. The filled symbols denote measurements obtained using noise introduced on the entire lattice and the open symbols are results from the Stochastic LapH approach. For every dilution scheme considered, Stochastic LapH significantly outperforms the lattice noise approach [9].

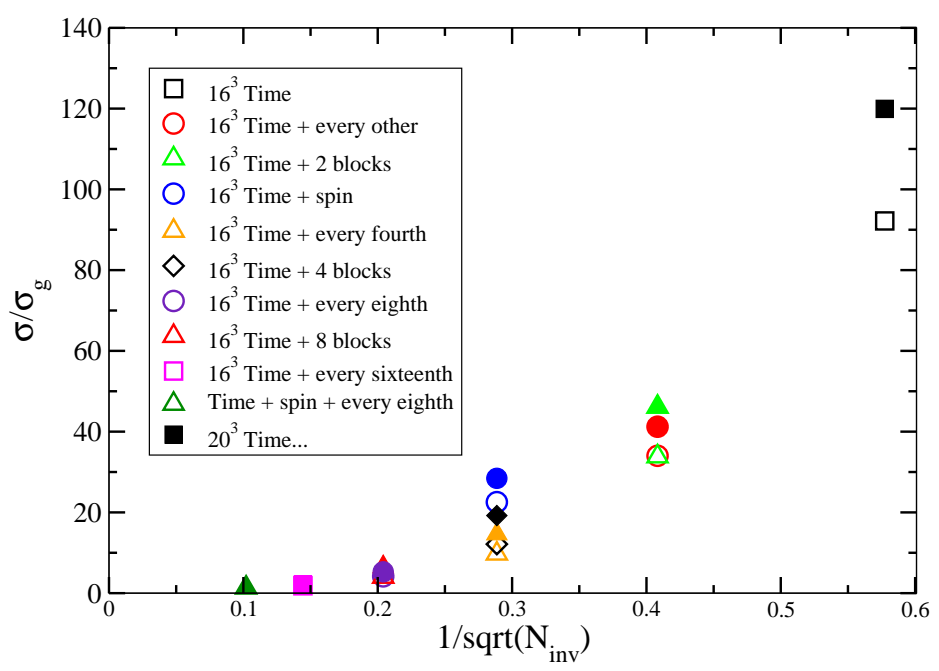

Figure 3: The statistical errors on a triply-displaced nucleon correlator at a source-sink separation of $5 a_{t}$ on two different lattice volumes. The correlator was computed using the stochastic smearing scheme with varying levels of dilution. The open symbols are results obtained on a $16^{3}$ spatial lattice volume, where the quark-smearing operator used 32 Laplacian eigenvectors. Closed symbols denote results on a $20^{3}$ spatial volume using 64 Laplacian eigenmodes. The growth in statistical error which comes from increasing the spatial volume while the smearing cutoff and the number of quark-matrix inversions per configuration are kept fixed is modest [9]. 


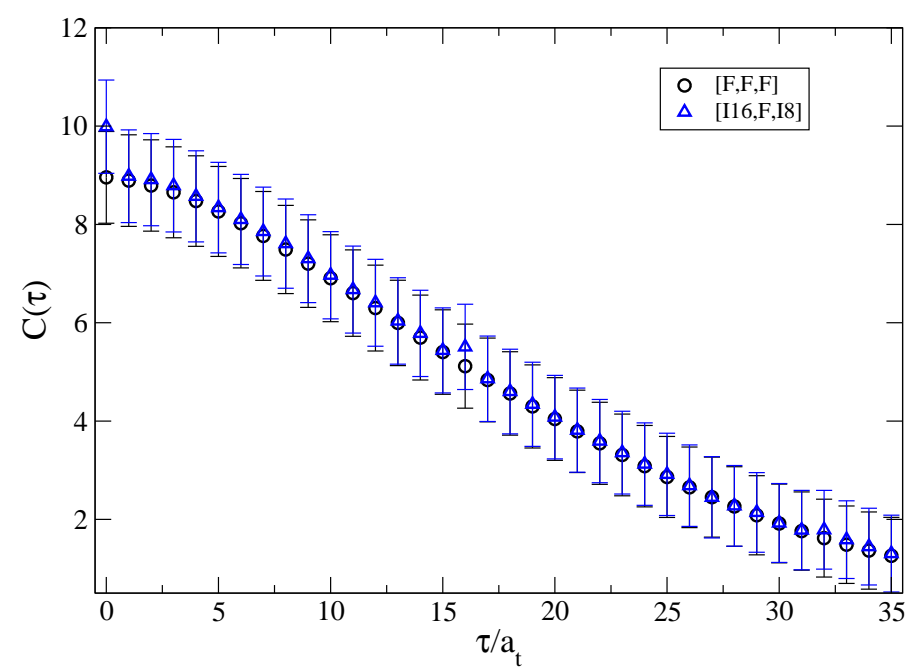

Figure 4: Disconnected contribution to an $I=0$ pseudoscalar correlator evaluated on $522+1$ flavor configurations on a $16^{3} \times 128$ lattice. The pion mass is approximately $400 \mathrm{MeV}$. The circles are results obtained using the exact LapH smearing scheme, and the triangular data points are Stochastic LapH estimates. The dilution scheme is interlaced 16 in time, full spin dilution, and interlace 8 in Laplacian-eigenmode space. Although the error estimates from both methods are similar, for this particular correlator, the stochastic approach requires a factor of 32 fewer quark matrix inversions than are needed in the exact scheme.

ment the exact scheme, the comparison could only be performed on the smaller $16^{3} \times 128$ lattice on a limited number of configurations. The exact method requires additional quark-matrix inversions for each sink time slice. However, the number of quark-matrix inversions needed for the stochastic estimate is drastically reduced by applying only partial time dilution to the quark lines at the sink. In that case, the optimal dilution schemes are expected to involve projectors which are interlaced in time.

Disregarding contact terms, the disconnected contributions to flavor-singlet meson correlators can be evaluated using a single diluted noise vector. We have computed the disconnected contributions to the isosinglet pseudoscalar and scalar correlators using the interpolating operators $\bar{\psi}_{l} \gamma_{5} \psi_{l}$ and $\bar{\psi}_{l} \psi_{l}$, respectively, where the subscript $l$ denotes a light quark flavor. Fig. 4 and Fig. 5 show results for the disconnected contributions. These results were obtained on 52 configurations and the correlators were averaged over 128 source time slices. The legends list the dilution schemes used in the quark-line estimates. [F,F,F] denotes full dilution in time, spin and eigenmode space, which corresponds to the exact smearing scheme. [I16,F,I8] indicates that dilution projectors are interlaced in time, with projectors containing every sixteenth time slice; the Stochastic LapH estimates employ full spin dilution, which was found to significantly reduce the variance in certain disconnected diagrams, and interlace eight eigenmode dilution. In both channels, the exact smearing scheme result and the dilute Stochastic LapH estimate have similar errors. However, the total number of quark-matrix inversions required for the exact estimate is $128 \times 4 \times 32=16384$, while the stochastic method involves just $16 \times 4 \times 8=512$ matrix inversions.

As an aside, the reader may have noticed a discrepancy between the exact and Stochastic LapH results for the contact term in Fig. 4, and, more obviously, in Fig. 5. This is due to the use of a 


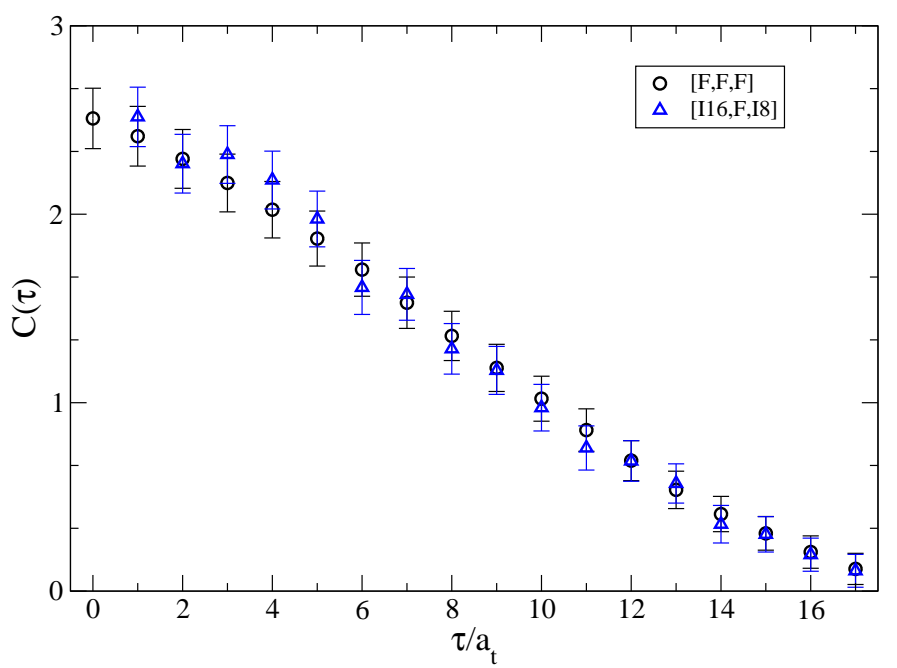

Figure 5: Disconnected contribution to an isosinglet scalar correlation function estimated using the exact and Stochastic LapH methods. The correlator shown was obtained after the subtraction of a large vev term. The discrepancy at $\tau=0$ arises because in the Stochastic LapH estimate a single diluted noise vector has been used for both the source and sink operators.

single noise vector for both source and sink operators, giving a biased correlator estimate when the temporal separation $\tau=0$, but having no effect on the measurement of spectral quantities.

Note that the scalar correlator shown in Fig. 5 was obtained after the subtraction of a large vacuum expectation value contribution. The quality of this signal, obtained on just 52 configurations, suggests that considerable progress can be made in the isosinglet meson sector using the Stochastic LapH method.

In the evaluation of disconnected diagrams, it is also possible to use different dilution schemes for the source and sink operators. One can, for example, use fully time-diluted noise vectors, borrowed from the calculation of the connected correlator component, to estimate the contracted source operator in the disconnected contribution. However, in this case, an average over all time slices is impractical. We tested this alternate estimate of the disconnected contribution, averaging the correlator over four randomly chosen source times. However, on a moderate number of configurations using a lower level of time dilution but averaging correlator estimates over all lattice time slices was found to give a cleaner signal, although the discrepancy between the estimates decreases on larger ensembles.

The inclusion of multi-hadron operators is a particular challenge for lattice hadron spectroscopy. Not only are the quark-line diagrams much more complicated, but we also need to evaluate correlators involving states whose constitutent hadrons have non-zero momenta.

The box diagram shown in Fig. 6 contributes to two-pion correlators in the $I=0,1$ sectors. The contribution of this diagram to an S-wave two-pion correlator measured on the $16^{3} \times 128$ lattice is displayed in Fig. 7. The interpolating operator used is simply the product of two zero-momentum single-pion interpolators. Once again, the circles denote results obtained using the exact smearing scheme, and the triangular data points are results from Stochastic LapH. In the stochastic scheme, 

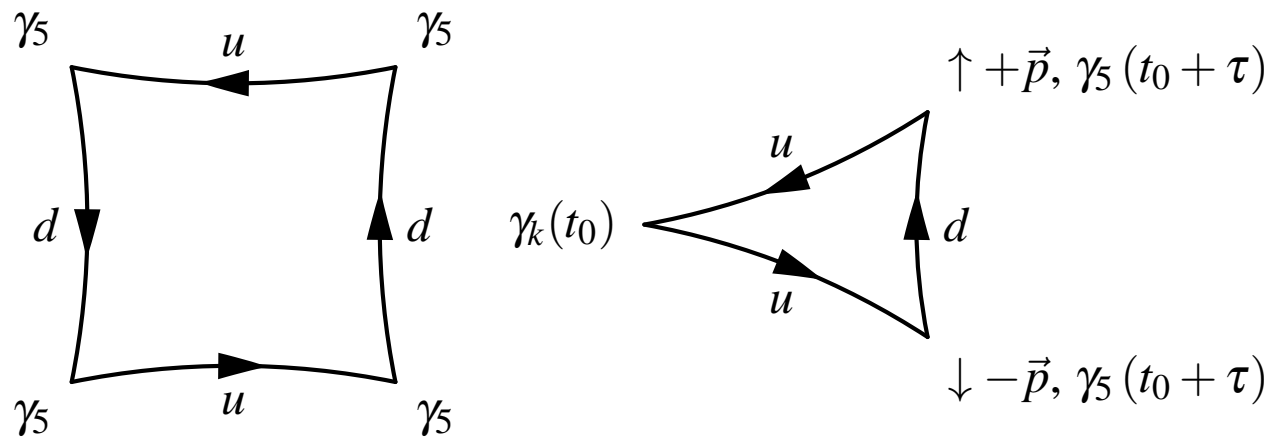

Figure 6: The two-pion box diagram and the rho-two-pion diagram discussed in the text.

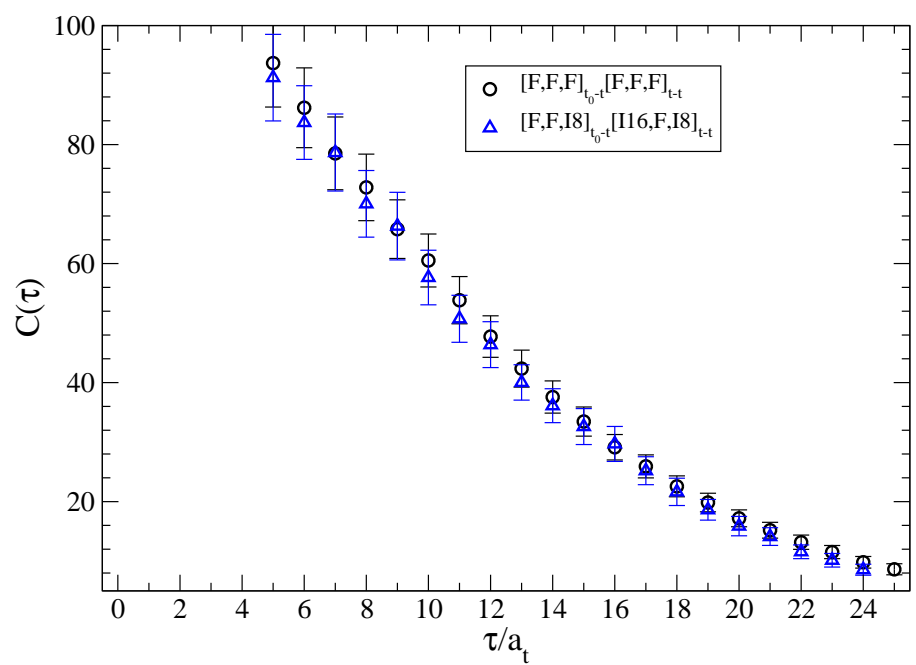

Figure 7: The box-diagram contribution to the S-wave, two-pion correlator evaluated with exact and Stochastic LapH. These results were again obtained on the $16^{3} \times 128$ lattice. The correlator has been averaged over 4 well-separated source time slices. The stochastic estimate uses just one diluted noise vector per quark line. In the stochastic scheme, quark lines connecting source and sink time slices are fully time dilute, while quark lines which begin and end on a single time slice use the interlaced 8 time dilution scheme. Once again, the results from the stochastic method are as accurate as results from the exact smearing scheme.

the quark lines connecting operator source and sink time slices are fully time diluted, while the quark lines which begin and end on a single time slice employ the interlaced 16 time dilution scheme. The stochastic estimate uses just one diluted noise vector per quark line. In both cases, the correlator estimates have been averaged over four source times.

In truth, Fig. 7 is not a fair comparison of the exact and Stochastic LapH schemes, since if it is feasible to apply the exact method, one can place source operators on all lattice time slices. However, on larger lattices the exact smearing scheme is prohibitively expensive, and Stochastic LapH is the only practical means of evaluating multi-hadron correlation functions. This figure does demonstrate the potential for obtaining accurate estimates for multi-hadron correlation functions using Stochastic LapH. A comparison of the error bars on the correlator estimates indicates that the variance in the Stochastic LapH estimate is dominated by gauge-field fluctuations, and no increase 


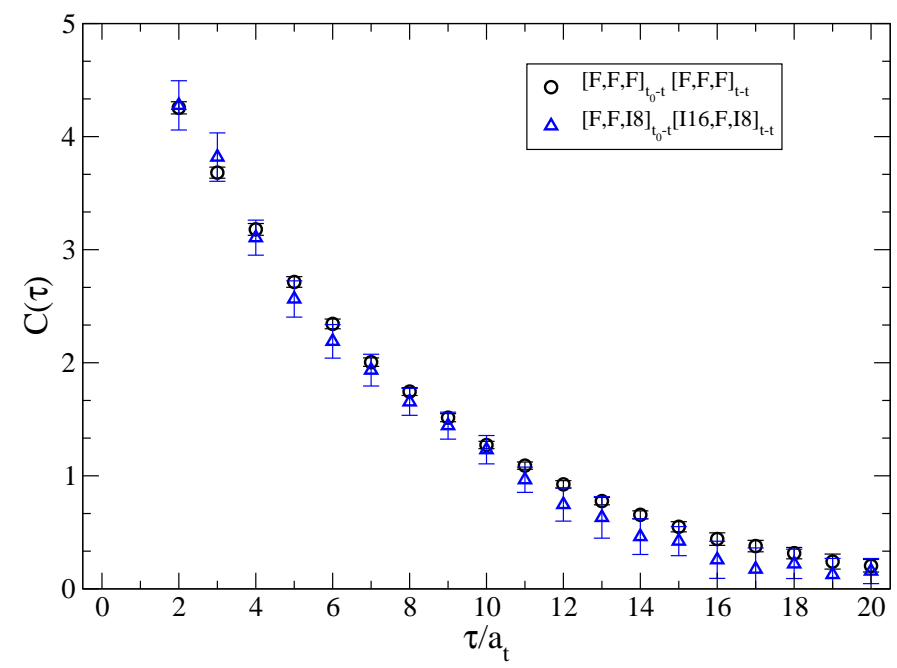

Figure 8: Two-point function correlating a $\bar{\psi} \gamma_{k} \psi$ source operator and a P-wave two-pion interpolator at the sink. In this case, the signal from Stochastic LapH is somewhat noisier than the exact method, although still reasonable. The exact method becomes computationally intractable on larger lattice volumes.

in the number of noise vectors or level of dilution is required.

As a final test of the Stochastic LapH method, we consider the two-point function correlating a $\rho$-meson operator at the source with and two-pion state at the sink. The $I=1$ two-pion system is in a P-wave state, which requires that the pions have non-zero back-to-back momenta. For the source operator, we use the simple quark bilinear: $\bar{\psi} \gamma_{k} \psi$. This operator is correlated with an interpolating operator for two pions with one unit of momentum each. As in the previous examples, the stochastic estimate uses a single noise vector per quark line. In this case, the Stochastic LapH algorithm gives a noticeably noisier result than exact smearing. However, the increase in error is moderate, and the stochastic result could be improved at negligible extra cost by averaging the correlator over the two possible ways of assigning noise vectors to the quark lines connecting source and sink time slices.

\section{Recent developments}

Motivated by the results from the $16^{3}$ lattice, we have recently begun a systematic study of the light hadron spectrum on a $24^{3} \times 128$ lattice ${ }^{2}$, using, in addition to single-hadron operators, interpolating operators for two-meson and meson-baryon states. To construct the required twohadron correlators, we need, for each valence quark mass, independent estimates for two quark lines that begin and end on a single time slice together with estimates for five quark lines connecting source and sink times. We use a single (diluted) noise vector per quark-line estimate, and employ the same dilution schemes that were applied to the disconnected and two-pion correlators on the $16^{3}$ lattice. Connected correlators have been averaged over four randomized source times. The number of quark matrix inversions needed to perform measurements using Stochastic LapH smearing is

\footnotetext{
${ }^{2}$ This corresponds to a spatial volume of approximately $(2.9 \mathrm{fm})^{3}$.
} 
then a factor of 34 times smaller than the number of inversions needed to implement the exact scheme.

The results obtained to date are promising. In particular, a comparison of isoscalar meson and two-pion correlators measured on the $24^{3}$ spatial volume with results from the $16^{3}$ lattice confirms that the mild volume dependence of the Stochastic LapH method in these systems. A detailed study of the Stochastic LapH algorithm on the $24^{3} \times 128$ lattice will be presented in a forthcoming publication [10].

\section{Summary and conclusions}

We presented a new algorithm for estimating hadron correlation functions involving smeared quark fields. Using Laplacian-Heaviside smearing, the number of quark matrix inversions needed to accurately estimate certain hadron correlators can be drastically reduced. However, the strong volume dependence of the smearing scheme means that an exact treatment of $S M^{-1} S$ is impractical on larger lattice volumes. We have outlined a way of implementing LapH smearing stochastically, using noise dilution to control the variance. Tests involving nucleon correlators show that the Stochastic LapH approach is more efficient than using dilute stochastic estimates for the quark propagators. Crucially, we have found that Stochastic LapH exhibits a mild volume dependence in this sector. The efficacy of this approach for disconnected correlators and multi-hadron correlators has also been demonstrated. On a small lattice volume, it was possible to compare the stochastic implementation of the LapH smearing scheme with the exact method. We found that Stochastic LapH gives results that are close to, or, in many cases, as accurate as the results obtained from the exact method, at a fraction of the computational cost. Finally, we noted that recent studies using Stochastic LapH performed on a larger lattice confirm excellent volume scaling for disconnected and multi-hadron correlators.

This algorithm is an exciting development for our spectroscopy program. It is now possible to include multi-hadron operators in our Monte Carlo measurements, and we can begin to extract resonance energies and widths from the measured finite-volume spectra [11, 12, 13]. Our results indicate that the isosinglet meson spectrum is also now accessible, although in that sector one also has to take potential mixing with glueball states into account. The challenges that remain to properly dilineate the low-lying hadron spectrum should not be underestimated, but, with continued technical and theoretical advances, considerable progress can be made toward this goal in the near future.

\subsection{Acknowledgements}

J.F. would like to acknowledge the hospitality of the Center for Computational Sciences and the Department of Physics at the University of Tsukuba. This work was supported by the U.S. National Science Foundation under awards PHY-0510020, PHY-0653315, PHY-0704171 and through TeraGrid resources provided by Athena at the National Institute for Computational Sciences (NICS) under grant number TG-PHY100027 and NICS and the Texas Advanced Computing Center under TG-MCA075017. M.P. is supported by Science Foundation Ireland under research grant 07/RFP/PHYF168. We thank our colleagues within the Hadron Spectrum Collaboration. Numerical calculations were performed using the Chroma software suite [14]. 


\section{References}

[1] J. Bulava et al, Excited-state nucleon spectrum with two flavors of dynamical fermions, Phys. Rev. D 79 (2009) 034505 [arXiv:0901.0027 [hep-lat]].

[2] S. Basak et al, Group-theoretical construction of extended baryon operators in lattice QCD, Phys. Rev. D 72 (2005) 094506.

[3] J. Dudek et al, Toward the excited meson spectrum of dynamical QCD, Phys. Rev. D 82 (2010) 034508 [arXiv: 1004.4930$]$.

[4] C. Morningstar et al, The excited hadron spectrum in lattice QCD using a new method of estimating quark propagation, talk given at Hadron 2009, [arXiv: 1002.0818].

[5] M. Peardon et al, A novel quark-field creation operator construction for hadronic physics in lattice QCD, Phys. Rev. D 80 (2009) 054506 [arXiv:0905.2160 [hep-lat]].

[6] C. Morningstar and M.J. Peardon Analytic smearing of SU(3) link variables in lattice QCD, Phys. Rev. D 69 (2004) 054501 [arXiv: 0311018]

[7] S. Basak et al, Combining quark and link smearing to improve extended baryon operators, PoS LAT2005 (2006) 076 [hep-lat/ 0509179$].$

[8] J. Foley et al, Practical all-to-all propagators for lattice QCD, Comput. Phys. Commun. 172 (2005) 145, [hep-lat/0505023].

[9] J. Bulava, An Improved Variance Reduction Technique for Stochastic All-to-All Quark Propagators in Lattice QCD Spectrum Computations, Ph.D. thesis at Carnegie Mellon University .

[10] C. Morningstar et al, Improved stochastic estimation of quark propagation with Laplacian Heaviside smearing in lattice $Q C D$, In preparation.

[11] P. Giudice et al, Extracting resonance parameters from lattice data, Proceedings of this conference, [arXiv:1009.6192 [hep-lat]].

[12] M. Lüscher, Signatures of unstable particles in finite volume. Nucl. Phys. B. 364 (1991) 237.

[13] V. Bernard et al, Resonance properties from the finite-volume energy spectrum, JHEP $\mathbf{0 8 0 8}$ (2008) 024 [arXiv:0806.4495 [hep-lat] ].

[14] R. Edwards and B. Joo, The Chroma software system for lattice QCD, Nucl. Phys. Proc. Suppl. 140 (2005) 832 [hep-lat/0409003]. 